\title{
Machine-vision-based Defect Detection Using Circular Hough Transform in Laser Welding
}

\author{
Qiao Ding ${ }^{1, *}$, Jianhua $\mathrm{Ji}^{1}$, Feng $\mathrm{Gao}^{2}$, Yatao Yang ${ }^{2}$ \\ ${ }^{1}$ Shenzhen Key Lab of Advanced Communication and Information Processing, Shenzhen \\ University, Shenzhen 518060, China; \\ ${ }^{2}$ Shenzhen Dade Laser Technology Co., Ltd., Shenzhen 518055, China. \\ *corresponding author: 972926257@qq.com
}

Keywords: laser welding, machine vision, Circular Hough Transform, defect detection

\begin{abstract}
In the field of laser welding, image processing technology is the general method to detect defects on the surface of the seam. Here the Hough Transform is introduced into the defect detection and the accuracy is greatly improved. The process is to use the industrial CCD(Chargecoupled Device) camera for image acquisition, then use Canny operator for edge detection, use Circular Hough Transform to locate the weld seam position, and finally compare the number of continuous pixels with the defect criteria to report detection results. Experimental data show that the false positive rate and false negative rate are greatly reduced after the Hough Transform is introduced into the defect detection, which has met the industrial requirements.
\end{abstract}

\section{Introduction}

Laser welding has been widely used for its advantage in narrowly focusing laser radiation to a small-area and high intensity heat source, which is instrumental in realizing deep penetration and high-speed welding and improved mechanical properties [1]. Vision-based defect detection on the surface of the seam is one of the methods to inspect laser welding quality. It can directly detect seam surface concavity, convexity, surface pores and other defects. It features high speed, real-time, high detection accuracy and is better than the other methods significantly in some applications. Here Circular Hough Transform is introduced into the defect detection. This method is more accurate and suitable for industrial production.

Hough Transform was first proposed by Hough Paul in 1962 [2], which can detect any analytic curve in the image space. The transformation from the image space to the parameter space is realized by the Hough transform, and an exact mathematical transformation formula is established. Initially it is applied to line detection, later has been widely used to detect circles, ellipses or even any shape curves. The main advantage of the Hough transform technique is that it is tolerant of gaps in boundary and is relatively unaffected by image noise [3], which can effectively filter out the noise to improve the accuracy of the results. For circles, the computational complexity and storage requirements are $\mathrm{O}\left(\mathrm{n}^{3}\right)$. If the radius of the circle is known, a power of the dimensionality of the circle can be reduced from $\mathrm{O}\left(\mathrm{n}^{3}\right)$ to $\mathrm{O}\left(\mathrm{n}^{2}\right)[4]$.

\section{Implementation}

The laser welding machine we built mainly consists of laser welding module, image acquisition module and defect detection module. Firstly, the product is automatically loaded by the machine and then transported to laser welding module through the guide rail. Secondly, the product is automatically welded by the fiber laser and then transported to image acquisition module through the guide rail. Thirdly, the image is obtained from the CCD camera and detected by the computer. Finally the product is transported through the guide rail and then uploaded by the machine. Its block diagram is shown in Fig. 1. 


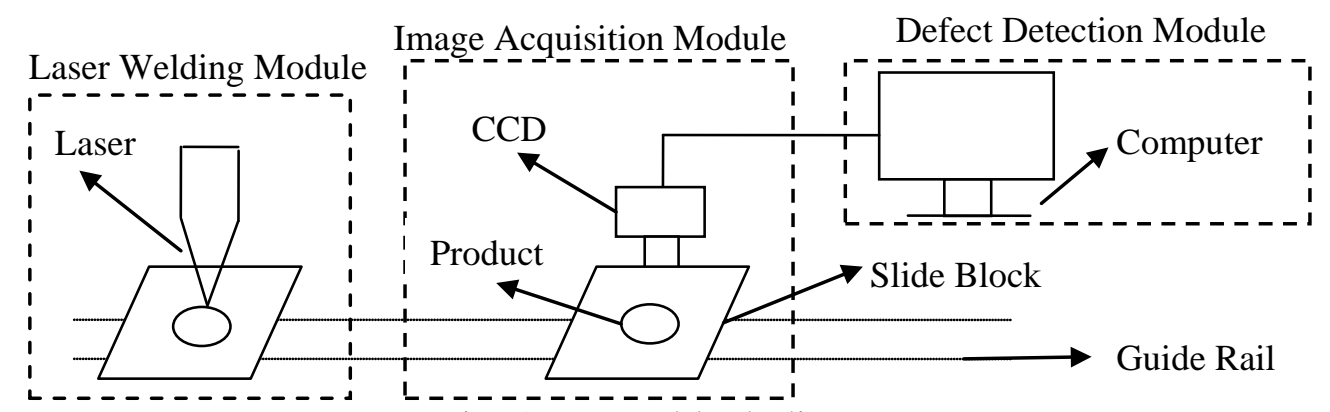

Fig. 1 system block diagram

\subsection{Image Processing}

Edge detection is an important topic in the research area of image analysis [5]. The purpose of edge detection in general is to significantly reduce the amount of data in an image, while preserving the structural properties to be used for further image processing.

After the image is captured by CCD camera, edge detection is prior to the defect detection for the image. Common edge detection methods are Sobel, Prewitt, Roberts, Canny [6] and so on. Canny has better anti noise performance, and can generate the edge of the gradient direction and intensity information, which provides convenience for the following processing.

Thus Canny is selected for edge detection in our system. The captured image is operated by Canny including the Gauss filter for smooth, first-order partial derivative and non maxima suppression of the derivative image.

\subsection{Circular Hough Transform}

The general equation of circle is as Eq. (1):

$$
(x-a)^{2}+(y-b)^{2}=r^{2}
$$

Where $(\mathrm{a}, \mathrm{b})$ is the circle center, and $\mathrm{r}$ is the radius. After mapping the circle from $\mathrm{x}-\mathrm{y}$ plane to a-b parameter space, the circle equation in parameter space is as Eq. (2):

$$
(a-x)^{2}+(b-y)^{2}=r^{2}
$$

The Hough transform needs to traverse (a, b, r) three parameters to describe each circle. For an image with known radius or radius range, it can be reduced from three-dimension to two-dimension and the traversal speed is faster. The objective becomes to find the $(\mathrm{a}, \mathrm{b})$ coordinates of the centers. Circular Hough Transform schematic diagram is shown in Fig. 2.

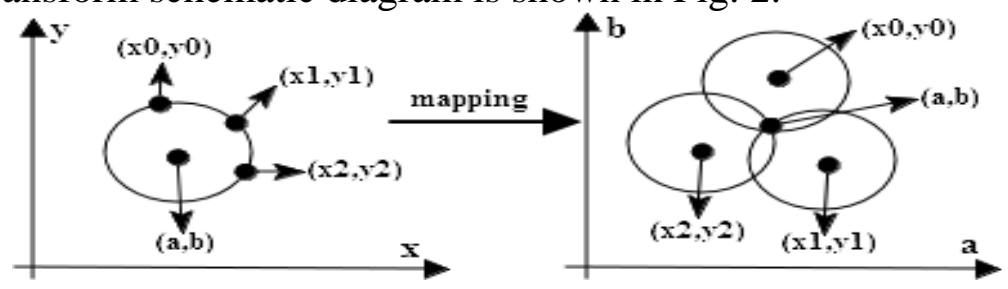

Fig. 2 Circular Hough Transform from the x-y plane (left) to the parameter space (right)

Circular Hough Transform is to traverse all the edge points of the x-y plane, and each point is mapped into a circle in the parameter space. Then count the number of the intersection of all circles, and the maximum number of points is the center of the circle. The center found means that the circle is found.

\subsection{Defect Detection}

For computer defect detection module, the flow chart is shown in Fig. 3. Firstly, the CCD camera transfers the collected image to the computer, and then the edge detection is performed on the collected images. Secondly, the position of weld seam is located by the Hough transform. It needs to use Hough Transform twice for locating the position. The first step is to locate the big circle and the second step is to locate two small circles inside the big circle. Finally the program calculates the number of continuous pixels near and inside the small circles. If the number exceeds the preset threshold, the product is a bad product; otherwise it is a good product. 


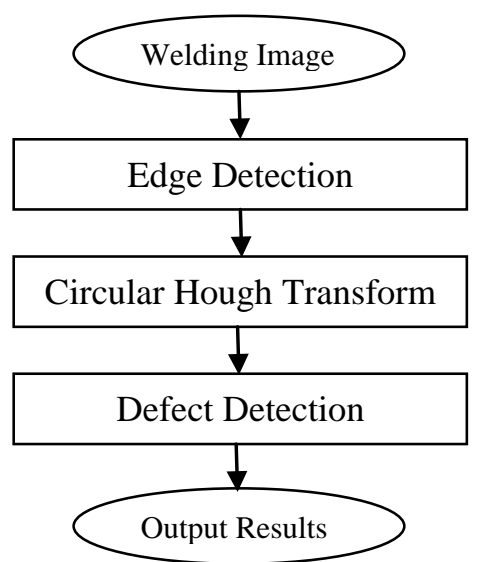

Fig. 3 defect detection flow chart

\section{Experimental Results}

The laser welding machine we built mainly uses IPG fiber laser and Basler camera. The development environment is Visual Studio 2012 with VB and C++ language to complete. Canny has two parameters, one is THRESH and another is SIGMA. THRESH is the sensitivity thresholds and SIGMA is the standard deviation of the Gaussian filter for the Canny method. Fig. 4 shows the original image. Fig. 5 to 6 shows the images after edge detection in different THRESH and SIGMA. And Fig. 6 only shows the inner part of the big circle. Fig. 7 is the result of Hough Transform for large circle detection. Fig. 8 is the result of Hough Transform for small circles detection.

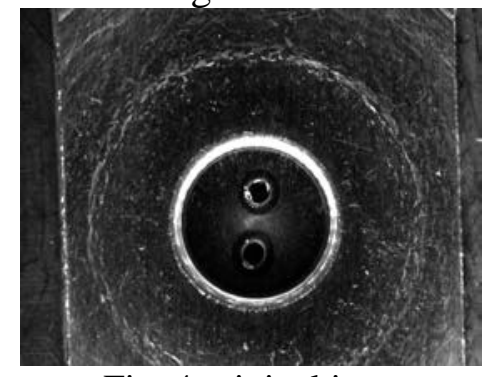

Fig. 4 original image

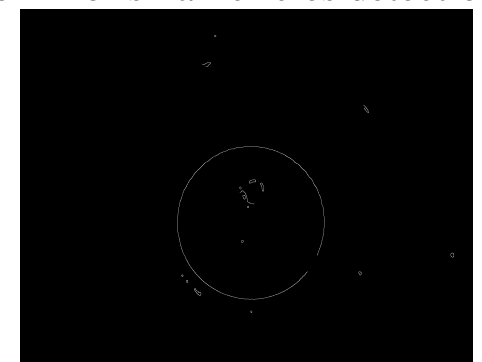

Fig. 5 image after canny edge detection

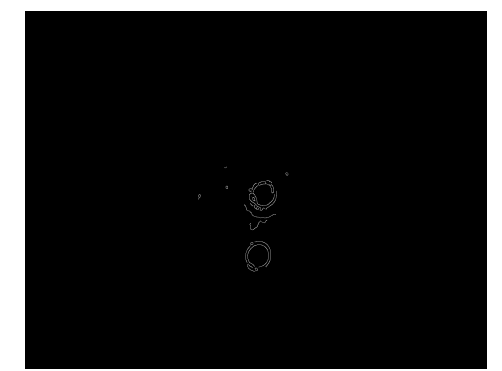

Fig. 6 image after canny edge detection in the inner big circle

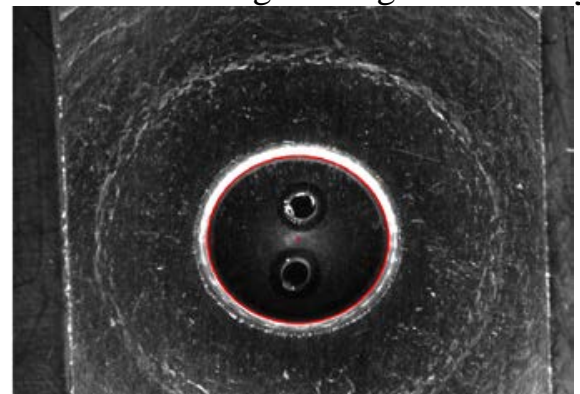

Fig. 7 Hough Transform for large circle detection

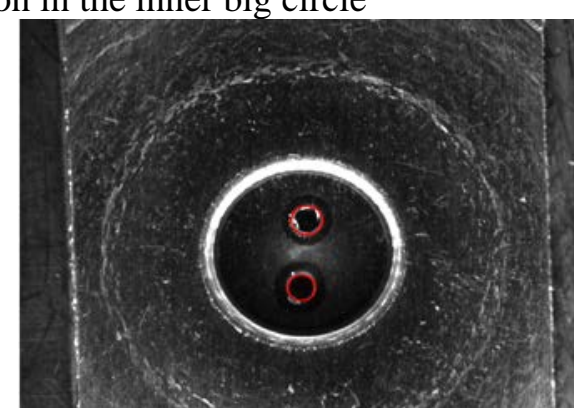

Fig. 8 Hough Transform for small circles detection

The false positive rate is the ratio of the quantity of false positive to the quantity of detection. The false negative rate is the ratio of the quantity of false negative to the quantity of detection. These two parameters are very important parameter to evaluate the accuracy of defect detection, 
especially the false negative rate. The general image processing method is to use template matching to find the circles. But this method is very inaccurate because of image noise influence. From table 1 it can be seen that the false positive rate is reduced to $2 \%$ and the false negative rate is reduced to $1 \%$ after the Hough transform is introduced into the defect detection. The accuracy rate is greatly improved.

Table 1 results of defect detection

\begin{tabular}{|c|c|c|c|}
\hline $\begin{array}{c}\text { Detection } \\
\text { Method }\end{array}$ & $\begin{array}{c}\text { Average number } \\
\text { of detection }\end{array}$ & $\begin{array}{c}\text { Average number of } \\
\text { false positive }\end{array}$ & $\begin{array}{c}\text { Average number of } \\
\text { false negative }\end{array}$ \\
\hline $\begin{array}{c}\text { General } \\
\text { image processing }\end{array}$ & 100 & 5 & 3 \\
\hline Hough Transform & 100 & 2 & 1 \\
\hline
\end{tabular}

\section{Conclusions}

The module which use Circular Hough Transform to detect the weld seam position first was simulated in the MATLAB, and then was converted to $\mathrm{C}++$ running on the machine. Experimental results show the Hough transform introduced into the defect detection based on machine vision is more accurate than general image processing techniques.

\section{References}

[1] S. Katayama and Y. Kawahito, Laser direct joining of metal and plastic, Scr. Mater., vol. 59, no. 12, pp. 1247-1250, Dec. 2008.

[2] Hough. P.V.C, Method and means for recognizing complex patterns, U. S. Patent, 4746348, 1962.

[3] Rafel C. Gonzalez and Richard E. Woods, Digital Image Processing (Second Edition), Publishing House of Electronics Industry, China, pp. 476-479, Sep. 2005.

[4] Y. Choe, H.-C. Lee, Y.-J. Kim, D.-H. Hong, S.-S. Park, and M.-T. Lim, Vision-based estimation of bolt-hole location using circular hough transform, in ICCAS-SICE, 2009, 18-21 2009, pp. 4821 4826.

[5] Soo-Chang Pei, Ji-Hwei Horng, Design of FIR bilevel Laplacian-of-Gaussian filter, (ELSEVIER) Signal Processing 82 (2002) 677-691.

[6] John Canny, A computational approach to edge detection, IEEE Trans. Pattern Anal. Mach. Intell., pp. 679-698, 1986. 